\title{
Screening asymptomatic patients with type 2 diabetes: The debates persist
}

\author{
George A. Beller, $M D{ }^{a}$ and Jamieson M. Bourque, $M D, M H S^{a}$ \\ ${ }^{a}$ Cardiovascular Division, Department of Medicine, University of Virginia Health System, \\ Charlottesville, VA
}

Received Aug 27, 2015; accepted Aug 27, 2015

doi: $10.1007 / \mathrm{s} 12350-015-0283-4$

During the past decade considerable discussion and debate pertaining to the clinical value and cost-effectiveness of imaging asymptomatic patients with Type 2 diabetes to detect functionally important coronary artery disease (CAD) have transpired. The controversy continues with two articles published in this issue of the Journal of Nuclear Cardiology. Petretta et $\mathrm{al}^{1}$ take the "Pro" position, whereas, Gibbons ${ }^{2}$ takes the "Con" position in this ongoing debate. These authors cite recent published clinical research studies to bolster their points of view.

The rationale for screening Type 2 diabetic patients for $\mathrm{CAD}$ stems from data indicating that $\mathrm{CAD}$ is the leading cause of mortality in these patients, accounting for $65 \%$ to $70 \%$ of deaths. ${ }^{3}$ Furthermore, these data showed that diabetes was associated with a 2 to 4 fold increased prevalence of $\mathrm{CAD}$, and an increased risk of dying from CAD, compared to nondiabetic patients. ${ }^{4,5}$ Other data supported the notion that patients with diabetes without a prior myocardial infarction (MI) had the same risk of coronary events as patients without diabetes and a prior MI. ${ }^{6}$ This led to the concept of diabetes as a coronary risk equivalent, which required more aggressive primary prevention measures such as statin therapy to reduce LDL cholesterol to below $100 \mathrm{mg} / \mathrm{dl}$. Since stress perfusion imaging was successful in separating high and low risk subsets of symptomatic patients, it was thought that if diabetes is a CAD equivalent, should not asymptomatic diabetic patients also undergo imaging to detect CAD and improve outcomes? The concept of $\mathrm{CAD}$ risk equivalency has been recently questioned following the report of a meta-analysis ${ }^{7}$ comprising more than 45,000 patients followed for a mean of

\footnotetext{
Reprint requests: Jamieson M. Bourque MD, MHS, Division of Cardiovascular Medicine, Department of Medicine, University of Virginia Health System, Charlottesville, VA; jbourque@virginia.edu J Nucl Cardiol 2015;22:1233-6.

$1071-3581 / \$ 34.00$

Copyright (C) 2015 American Society of Nuclear Cardiology.
}

13.4 years. This analysis showed, contrary to the existing paradigm, that patients with diabetes and no prior MI had actually a $43 \%$ lower risk of future CAD events compared with patients without diabetes and a prior MI. This finding and the observation that one-third of asymptomatic diabetic patients have no evidence of coronary atherosclerosis by CTA or CAC scoring ${ }^{8}$ have led to movement away from considering Type 2 diabetes as a CAD equivalent.

It should be noted that published studies reporting the percentage of asymptomatic Type 2 diabetic patients that have significant CAD have patient populations with substantial differences in clinical risk profiles. Some of these diabetic patient populations, despite being asymptomatic at the time of imaging, have a significant number of patients with either known CAD, peripheral vascular disease, renal disease, or an abnormal resting ECG. These may have been symptomatic in the past and even may have had a prior MI or revascularization. For example, in the Mayo Clinic cohort of asymptomatic diabetic patients, $50 \%$ were referred for preoperative risk assessment, $43 \%$ had ECG Q-waves and 28\% had peripheral vascular disease. ${ }^{9}$ In contrast, other asymptomatic patient cohorts in published studies were at lower clinical risk, with no prior history of $\mathrm{CAD}$, no prior symptoms consistent with $\mathrm{CAD}$, and a normal resting electrocardiogram. An example of this type of population is the DIAD study ${ }^{10}$ which excluded patients with a history of CAD or those with an abnormal resting ECG. Both types of asymptomatic diabetic patient cohorts are included in screening studies, although the probability of detecting $\mathrm{CAD}$, and determining an increased risk for future cardiac events, are higher in the former. This should be kept in mind when reviewing reports either supporting or negating the concept of screening for CAD in diabetic patients. Other issues regarding the value of screening raised by Dr. Gibbons in his argument against routine screening of asymptomatic patients is whether it provides incremental 
prognostic information to traditional CAD risk factors, and whether it will lead to changes in treatment that improves outcomes compared to standard guideline medical therapy and other primary prevention measures (e.g., invasive strategies for silent ischemia, even greater reduction of LDL cholesterol, etc.). To our knowledge, these benefits of screening have not yet been demonstrated. Certainly, knowledge of presence of occult coronary atherosclerosis or significant CAD found by screening might lead to better compliance with medical therapy, and better outcomes. This also has not been definitively demonstrated.

It has been observed that the prevalence of high-risk $\mathrm{CAD}$ and ischemia on stress imaging in diabetic patients may be decreasing, presumably because of both effective primary and secondary prevention. ${ }^{11}$ This correlates well with the overall decrease in significant inducible ischemia seen in patients referred for stress SPECT imaging. ${ }^{12}$ Thus, if the prevalence of CAD and high-risk ischemia is decreasing in the Type 2 diabetic population, then screening for CAD and ischemia in such patients adequately treated with statins, ACE inhibitors and adhering to lifestyle changes, would most likely not yield a high percentage of patients for whom a subsequent invasive strategy would be effective in reducing risk of future cardiac events. If that is the case, then optimal medical therapy alone for this population would be the most cost-effective approach to management. Park et al performed coronary computed tomography angiography (CTA) and coronary artery calcium (CAC) imaging in more than 2000 asymptomatic subjects. ${ }^{13}$ Diabetic patients were matched by propensity scoring to nondiabetic patients. No significant differences between the matched pairs in the prevalence of $\geq 50 \%$ stenosis of the left main or proximal left anterior descending artery (5.3\% vs $3.8 \%$ ), multivessel disease $(4.4 \%$ vs $2.9 \%)$, or high-risk CAD (4.3\% vs $2.7 \%)$ was observed. Similarly, there was no significant difference in the composite of all-cause death, MI, acute coronary syndrome and coronary revascularization between the two groups. Thus, no differences in high-risk CAD and adverse outcomes were seen between asymptomatic diabetic and asymptomatic nondiabetic subjects in this study. The prevalence of high-risk CAD was quite low, reflecting the population of asymptomatic diabetic subjects who had no prior history of angina, MI, revascularization or an abnormal ECG.

Bourque et $\mathrm{al}^{11}$ prospectively evaluated 575 consecutive diabetic patients (29\% asymptomatic) who underwent stress SPECT myocardial perfusion imaging. Ischemia of $\geq 10 \%$ of the left ventricle was seen in only $5.0 \%$ of the patients imaged. Fewer than $1 \%$ had early revascularization within 12 weeks of the study. At a median of 4.4 years of follow-up, the rate of cardiac death was only $0.8 \%$ per year. One-third of the patients with events had a normal myocardial perfusion scan. There was no difference in events between symptomatic and asymptomatic patients. Fifty-eight percent of the asymptomatic diabetic patients achieved $\geq 10$ METS of workload. Interestingly, $43 \%$ of the asymptomatic patients had known CAD, and yet the event rate was extremely low. This may, in part, be attributed to the observation that more than $50 \%$ of these patients were on statins, ACE inhibitors, aspirin and beta blockers. These findings of a low event rate in patients with Type 2 diabetes is consistent with the results of the BARI 2D trial, in which the survival rate at 5 years for the medically treated group was $87.8 \% .^{14}$ Thus, as suggested in the CTA study cited previously by Park et al, ${ }^{13}$ the study by Bourque et $\mathrm{al}^{11}$ shows a decreasing prevalence of high-risk SPECT ischemia and a lower cardiac mortality in diabetic patients in the current era. The other finding of the study of Bourque et $\mathrm{al}^{11}$ worthy of mention, is that an initially low cardiac event rate in patients without scintigraphic ischemia increased after 2 years. This suggests a shorter warranty period for a low event rate in diabetic patients with no inducible ischemia on MPI. This may be a limitation for routine screening with MPI in this patient population to identify a low risk subset with a good long-term event-free survival.

Gibbons cites both the DIAD ${ }^{10}$ and FACTOR- $64^{15}$ randomized studies performed in asymptomatic diabetic patients to support his position against routine screening with cardiac imaging. Neither study found differences in outcomes between patients randomized to screening or to routine care. The event rates were low in both studies. In the DIAD study, at 5 years of follow-up, the event rate was $2.7 \%$ in the screening group and $3.0 \%$ in the medical treatment group. In FACTOR-64, in which CTA was the imaging modality, the event rate at about 4 years, was $6.2 \%$ in the screening group and $7.6 \%$ in the medical treatment group. These event rates were lower than expected. In this study, only $11 \%$ of patients had severe stenosis. The mean LDL cholesterol in patients enrolled in FACTOR 64 was $87.7 \mathrm{mg} / \mathrm{dl}$. Gibbons mentions that using FACTOR-64 data, 112 patients would need to be screened by CTA to identify one patient with severe proximal CAD. In DIAD, only $6 \%$ of the asymptomatic patients had moderate-severe defects. These patients did have an increase in events compared to the other patients imaged in this study. However, the overall event rates for the screened group were similar to the non-screened group.

Petratta et $\mathrm{al}^{1}$ who took the Pro position for screening, propose that "detection of occult CAD might allow the initiation of appropriate therapy at a time point when the disease process is more easily modifiable. ${ }^{1,}$ They quote the IDIS trial of high-risk diabetic patients, 
many of whom were symptomatic or had a prior MI, in which the addition of myocardial perfusion imaging improved risk classification over traditional risk factors and ECG stress test data. ${ }^{16}$ No one would dispute that the addition of imaging is expected to provide supplemental prognostic information in this population of patients. However, the conclusions can't be applied to a lower risk group of asymptomatic patients, with a substantially lower pretest risk of significant CAD. Petretta et $\mathrm{al}^{1}$ superbly summarize other observational studies in asymptomatic diabetic patient populations, including their own, showing that silent ischemia can be detected, and that such ischemic findings are associated with an increased risk of cardiac events. Again, such observational studies do not shed light on whether outcomes are any better than optimal medical therapy, particularly in lower risk asymptomatic patients with no prior CAD, a normal resting ECG, no peripheral vascular disease, and no chronic renal disease. These are the type of patients that are the focus of the debate and controversy.

Petratta et $\mathrm{al}^{1}$ cite the more recent Basel Asymptomatic High-Risk Diabetes Outcome (BARDOT) trial, ${ }^{17}$ which prospectively enrolled 400 Type 2 diabetic patients with no history or symptoms of CAD, but who were at high risk because of either vascular disease, autonomic neuropathy, nephropathy, retinopathy, microalbuminuria, or a diabetes duration of $>5$ years with two or more CAD risk factors. All patients had two SPECT studies 2 years apart. Baseline SPECT results showed an abnormality in $22 \%$ of the patients. Patients with an abnormal SPECT were randomized to revascularization with medical therapy $(n=43)$ or to medical therapy alone $(n=39)$. Although these numbers are small, the development of new ischemia or scar, and the number of patients with "overt or silent CAD progression" was greater in patients randomized to the medical strategy. No difference in hard event rates was seen at 2 years of follow-up. The $80 \%$ of the patients with a normal scan had only a $0.7 \%$ cardiac death rate and $1.0 \%$ MI rate with a very low prevalence of scintigraphic progression of CAD (4.6\%) at 2 years. Again, it should be pointed out that this was a clinically high-risk cohort of asymptomatic diabetic patients. Yet, only $1 / 5$ th of the SPECT studies were abnormal, and only 12 of the 400 patients imaged at baseline had a summed stress score of $\geq 10 \%$ of the LV.

Another approach to refine risk stratification in intermediate risk asymptomatic diabetic patients is to perform CAC imaging, ${ }^{18}{ }^{20}$ which is less expensive than SPECT MPI and has less radiation exposure. Studies have shown that Type 2 diabetic patients with a high CAC score have a higher prevalence of ischemia by SPECT compared to patients with a low CAC score or no $\mathrm{CAC}^{21}$ and have a worse outcome. Higher CAC scores correlate with greater event rates. Whether or not it is appropriate to use the CAC score as a gateway to SPECT imaging, or to justify a more aggressive management approach over optimal medical therapy alone to improve outcomes, is not known. Patients at low risk for future cardiac events can also be identified by CAC imaging since 1/3rd of all asymptomatic diabetic patients have a CAC score of $<10$. A finding of a CAC score of 0 might permit a less intensive approach to medical therapy. In the 2010 American College of Cardiology/American Heart Association guideline for the assessment of cardiovascular risk in asymptomatic adults, ${ }^{22}$ the recommendation for patients with diabetes indicated that, "In asymptomatic adults with diabetes, 40 years of age and older, measurement of CAC is reasonable for cardiovascular risk assessment." This received a Class IIa recommendation. Furthermore, the guideline stated that stress MPI can be considered for advanced cardiovascular risk assessment when previous risk assessment testing suggests a high risk of CAD, "such as a CAC score of 400 or greater." This received a Class IIb recommendation. ${ }^{22}$

In summary, the weight of the evidence to date, particularly from the randomized studies cited above, indicate that routine screening of all asymptomatic Type 2 diabetic patients with either MPI or CTA cannot be recommended. No data are yet available showing that outcomes are favorably changed employing such a routine screening approach. This does not mean that if imaging was performed it would not identify patients with silent ischemia or occult CAD as Petretta et $\mathrm{al}^{1}$ declare. The DIAD study ${ }^{10}$ identified a patient subset with high-risk scans and an increased event rate. However, the event rate was no different from what was observed in the non-screened group. This is because high-risk patients were still identified in the nonscreened group and underwent revascularization, but not prompted by routine SPECT imaging. Finally, with current primary prevention medical therapy in Type 2 diabetic patients, it can be expected that the prevalence of high-risk CAD, inducible ischemia and hard cardiac events will continue to decrease. This has already been noted in some of the studies cited previously in this editorial. That makes an expensive screening strategy with radiation exposure in the asymptomatic patient even less appealing. Nevertheless, we would advocate continued clinical research in this area, particularly focused on randomizing asymptomatic Type 2 diabetic patients to a CAC imaging strategy versus a standard primary prevention strategy with guideline-based medical therapy. We congratulate Dr. Gibbons and Dr. Petretta and colleagues for continuing to bring this controversy to the forefront for thought and discussion. We predict that this debate regarding the worth of 
screening asymptomatic diabetic patients with an imaging technique will persist until definitive randomized trials powered for hard cardiac events and with economic analyses are undertaken. In addition, more advanced imaging technologies such as positron emission tomography (PET) and cardiac magnetic resonance (CMR) are being investigated as modalities to better risk stratify patients. For example, myocardial flow reserve as measured by quantitative PET may be superior for risk assessment in diabetic patients compared to solely detecting relative focal defects. ${ }^{23}$ It is gratifying to see that clinical research in this area is still quite robust.

\section{Disclosure}

Dr. Bourque receives research grant support from Astellas Global Development Inc. Dr. Beller has nothing to disclose.

\section{References}

1. Petretta M, Cuocolo A. Screening asymptomatic patients with type 2 diabetes is recommended: Pro. J Nucl Cardiol 2015. doi: 10.1007/s12350-015-0250-0.

2. Gibbons RJ. Screening asymptomatic patients with type 2 diabetes is recommended-Con. J Nucl Cardiol 2015. doi:10.1007/ s12350-015-0248-7.

3. Mozaffarian D, Benjamin EJ, Go AS, Arnett DK, Blaha MJ, et al. Heart Dis Stroke Stat 2015;131:e1-295.

4. Sarwar N, Gao P, Seshasai SR, et al. Diabetes mellitus, fasting blood glucose concentration, and risk of vascular disease: A collaborative meta-analysis of 102 prospective studies. Lancet 2010; 375:2215-22.

5. Kannel WB, McGee DL. Diabetes and glucose tolerance as risk factors for cardiovascular disease: The Framingham study. Diabetes Care 1998;21:1138-45.

6. Haffner SM, Lehto S, Ronemaa T, Pyorala K, Laakso M. Mortality from coronary heart disease in subjects with type 2 diabetes and in nondiabetic subjects with and without prior myocardial infarction. N Engl J Med 1998;339:229-34.

7. Bulugahapitiya U, Siyambalapitiya S, Sithole J, Idris I. Is diabetes a coronary risk equivalent? Systematic review and meta-analysis. Diabetes Med 2009;26:142-8.

8. Malik S, Budoff MJ, Katz R, Blumenthal RS, Bertoni AG, Nasir $\mathrm{K}$, et al. Impact of subclinical atherosclerosis on cardiovascular disease events in individuals with metabolic syndrome and diabetes: The multiethnic study of atherosclerosis. Diabetes Care 2011;34:2285-90.

9. Rajagopalan N, Miller TD, Hodge DO, Fye RL, Gibbons RJ. Identifying high-risk asymptomatic diabetic patients who are candidates for screening stress single-photon emission computed tomography imaging. J Am Coll Cardiol 2005;45:43-9.

10. Young LH, Wackers FJTh, Chyun DA, Davey JA, Barrett EJ, Taillefer R, et al. Cardiac outcomes after screening for asymptomatic coronary artery disease in patients with type 2 diabetes:
The DIAD study: A randomized controlled trial. JAMA 2009;301:1547-55.

11. Bourque JM, Patel CA, Ali MM, Perez M, Watson DD, Beller GA. Prevalence and predictors of ischemia and outcomes in outpatients with diabetes mellitus referred for single-photon emission computed tomography myocardial perfusion imaging. Circ Cardiovasc Imaging 2013;6:466-77.

12. Rozanski A, Gransar H, Hayes SW, Min J, Friedman JD, Thomson LE et al. Temporal trends in the frequency of inducible ischemia during cardiac stress testing: 1991-2009. J Am Coll Cardiol 2013;61:1054-65.

13. Park G-M, Lee J-H, Lee S-W, Yun S-C, Kim Y-H, Cho Y-R, et al. Comparison of coronary computed tomographic angiographic findings in asymptomatic subjects with versus without diabetes mellitus. Am J Cardiol 2015;116:372-8.

14. The Bari 2D Study Group. A randomized trial of therapies for type 2 diabetes and coronary artery disease. N Engl J Med 2009;360: 2503-15.

15. Muhlestein JB, Lapppe DL, Lima JAC. Effect of screening for coronary artery disease using CT angiography on mortality and cardiac events in high-risk patients with diabetes: The FACTOR64 randomized clinical trial. JAMA 2014;312:2234-43.

16. Acampa W, Petretta M, Evangelista L, Daniele S, Xhoxhi E, De Rimini ML, et al. Myocardial perfusion imaging and risk classification for coronary heart disease in diabetic patients. The IDIS study: A prospective, multicenter trial. Eur J Nucl Med Mol Imaging 2012;39:387-95.

17. Zellweger MJ, Maraun MD, Osterhues HH, Keller U, MullerBrand J, Jeger R, et al. Progression to overt or silent CAD in asymptomatic patients with diabetes mellitus at high coronary risk: Main findings of the prospective multicenter BARDOT trial with a pilot randomized treatment substudy. J Am Coll Cardiol 2014;7:1001-10.

18. Raggi P, Shaw LJ, Berman DS, Callister TQ. Prognostic value of coronary artery calcium screening in subjects with and without diabetes. J Am Coll Cardiol 2004;43:1663-9.

19. Elkeles RS, Godsland IF, Feher MD, Rubens MB, Roughton M, Nugara $\mathrm{F}$, et al. Coronary calcium measurement improves prediction of cardiovascular events in asymptomatic patients with type 2 diabetes: The PREDICT study group. Eur Heart J 2008;29: 2244-51.

20. Hecht HS. Coronary artery calcium scanning; past, present and future. JACC Cardiovasc Imaging 2015;8:579-96.

21. Anand DV, Lim E, Hopkins D, Corder R, Shaw LJ, Sharp P et al. Risk stratification in uncomplicated type 2 diabetes: Prospective evaluation of the combined use of coronary artery calcium imaging and selective myocardial perfusion scintigraphy. Eur Heart $\mathbf{J}$ 2006;27:713-21.

22. Greenland P, Alpert JS, Beller GA, Benjamin EJ, Budoff MJ, Fayad ZA, et al. 2010 ACCF/AHA guideline for the assessment of cardiovascular risk in asymptomatic adults: Executive summary: A report of the American College of Cardiology Foundation/ American Heart Association task force on practice guidelines. $\mathrm{J}$ Am Coll Cardiol 2010;56:2182-99.

23. Murthy VL, Masano N, Foster CR, Gaber M, Hainer J, Dorbala S, et al. Association between coronary vascular dysfunction and cardiac mortality in patients with and without diabetes mellitus. Circulation 2012;126:1858-68. 